\title{
Optical generation of pure spin currents at the indirect gap of bulk $\mathrm{Si}$
}

\author{
F. Bottegoni ${ }^{*}$ C. Zucchetti, F. Ciccacci, M. Finazzi, and G. Isella \\ LNESS-Dipartimento di Fisica, Politecnico di Milano, \\ Piazza Leonardo da Vinci 32, 20133 Milano, Italy
}

(Dated: July 24, 2016)

\begin{abstract}
We report on the optical generation of a pure spin current at the indirect gap of bulk Si at room temperature. This is experimentally addressed by exploiting a $\mathrm{Pt} / \mathrm{n}$-doped $\mathrm{Si}(001)$ junction: in this case circularly-polarized light promotes spin-polarized electrons to the $\Delta$ valleys of the Si Brillouin zone. These electrons then diffuse toward the $\mathrm{Pt} / \mathrm{Si}$ interface and enter the $\mathrm{Pt}$ layer, where the spin accumulation is detected through the inverse spin-Hall effect (ISHE). We characterize the photon energy dependence of the ISHE signal, which can be accurately described in the frame of a onedimensional spin drift-diffusion model and estimate the electron spin lifetime $\tau_{\mathrm{s}}$ in bulk Si to be $\tau_{\mathrm{s}}=15 \pm 5 \mathrm{~ns}$.
\end{abstract}

One of the most important goals of spintronics is to combine electronic and spin degrees of freedom of carriers on the Si platform to handle classical or quantum information. In this frame optical spin orientation, i.e the possibility of optically exciting spin-polarized electrons by means of circularly-polarized light [1], is a powerful tool to explore spin transport and dynamics in semiconductors 22. The optical injection of spin-oriented electrons was first achieved by Lampel in Si at low temperature [3. However, after these pioneering studies, the low spin-orbit interaction and the long carrier lifetime in bulk Si 4, 5] have prevented further investigations of optical spin injection in $\mathrm{Si}$ and optical orientation studies have been almost completely focused on III-V semiconductors [6] 8], with a few reports on Ge 9] and, more recently, on SiGe heterostructures [10].

In this process, dipole selection rules for optical transitions with circularly-polarized light allows for the generation of a spin-oriented electron population in the conduction band of the semiconductor with a spin polarization $P=\left(n_{\uparrow}-n_{\downarrow}\right) /\left(n_{\uparrow}+n_{\downarrow}\right)$, being $n_{\uparrow(\downarrow)}$ the up-(down-) spin densities referred to the quantization axis given by the direction of light propagation in the material. Spinorbit interaction plays a key role: both in GaAs and Ge, the energy separation between heavy hole ( $\mathrm{HH})$-light hole $(\mathrm{LH})$ and split-off $(\mathrm{SO})$ states in the valence band at $\Gamma$ is $\Delta E_{\mathrm{SO}} \approx 300 \mathrm{meV}$, so that the incident photon energy can be easily tuned to promote in the conduction band only electrons coming from $\mathrm{HH}$ and $\mathrm{LH}$ states. As a consequence, in bulk GaAs- and Ge-based semiconductors spin polarization values approaching $P \approx 50 \%$ can be achieved.

In bulk $\mathrm{Si}$, the scenario is radically different: the direct bandgap at $\Gamma$ lies in the ultraviolet range $\left(E_{\mathrm{dg}}=4.2\right.$ $\mathrm{eV}$ at room temperature) and the energy difference between HH-LH and SO states is only $\Delta E_{\mathrm{SO}} \approx 40 \mathrm{meV}$, whereas the fundamental gap is indirect $\left(E_{\mathrm{ig}}=1.12 \mathrm{eV}\right.$ at room temperature). Nevertheless, theoretical calculations indicate that phonon-assisted optical transitions at the indirect gap of $\mathrm{Si}$ could generate a spin-oriented electron population with $P \approx 5 \%[11,12$ and indeed ex- perimental evidence of optical spin orientation in Si have been obtained but only at cryogenic temperatures $[3]$ and with the use of fairly large magnetic fields [13].

In recent years the exploitation of electrical spininjection schemes, both at low [14-19] and room temperature [20-23] and the prediction of large intrinsic electron spin lifetimes [24 29] have renewed the interest of the spintronic community for bulk Si and Si-based heterostructures [26. In this framework the exploitation of optical orientation in $\mathrm{Si}$ would add a relevant building block for the design of spintronic devices and potentially lead to the integration of electronic, photonic and spintronic functionalities on a single material platform.

In this paper, we report on the optical generation of a pure spin current at the indirect gap of bulk $\mathrm{Si}(001)$ at room temperature. To this purpose we have combined optical orientation with an electrical detection scheme based on the inverse spin Hall effect (ISHE) [30, which takes place within a Pt layer forming a Schottky junction with the investigated sample. Such a photo-induced ISHE approach have been already used to detect optically excited spins at the direct gap of GaAs [31 33], InP 34] and Ge [35.37.

The photo-induced ISHE signal has been measured in a $\mathrm{Pt} / \mathrm{n}$-doped $\mathrm{Si}$ junction: the spin-oriented electrons, generated at the $\Delta$ valleys of bulk $\mathrm{Si}$, diffuse toward the $\mathrm{Pt} / \mathrm{Si}$ interface and yield an electromotive force at the edges of the thin Pt layer through spin-dependent scattering with $\mathrm{Pt}$ nuclei. We have also investigated the photon energy dependence of the ISHE signal in the range between $1.2<h \nu<1.8 \mathrm{eV}$ and we have interpreted the experimental results by means of a drift-diffusion model [33] which fairly reproduces the main spectral features, and allows for an estimation of the electron spin lifetime at the $\Delta$ minima giving $\tau_{\mathrm{s}}=15 \pm 5 \mathrm{~ns}$.

The investigated device is depicted in Fig. 1 13 , together with the experimental geometry: a uniform $l_{\mathrm{x}} \times l_{\mathrm{y}}=5 \times 5$ $\mathrm{mm}^{2}$-wide and $4 \mathrm{~nm}$-thick Pt layer is deposited by ebeam evaporation on top of a $500 \mu \mathrm{m}$-thick phosphorousdoped $\operatorname{Si}(001)$ substrate $\left(\rho_{\mathrm{Si}} \approx 5 \Omega \cdot \mathrm{cm}\right.$, doping concentration $\left.N_{\mathrm{P}} \approx 8.95 \times 10^{14} \mathrm{~cm}^{-3}\right)$. Two $200 \mathrm{~nm}$-thick 

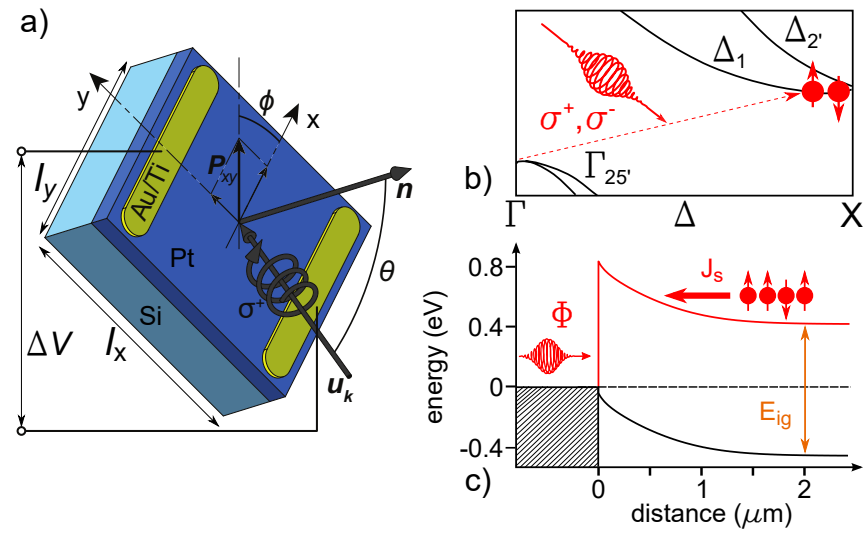

FIG. 1. (Color online) (a) Scheme of the Pt/Si sample and the experimental geometry: $\theta$ is the angle between the direction of the incident light $\mathbf{u}_{\mathrm{k}}$ and the normal $\mathbf{n}$ to the sample surface, whereas $\varphi$ is the angle between the projection of $\mathbf{u}_{\mathrm{k}}$ in the sample plane and the $x$ axis. (b) Sketch of the $\Gamma \rightarrow \Delta$ indirect transitions involved in the optical orientation process. The symmetry of the electronic states according to the Koster notation [38] is reported. (c) Pt/Si Schottky junction and band alignment at the $\mathrm{Pt} / \mathrm{Si}$ interface under illumination with a photon flux $\Phi$ and open-circuit conditions. The measured Schottky barrier height is $E_{\mathrm{B}}=0.85 \mathrm{eV}$, in good agreement with reported values of Ref. 39

$\mathrm{Au} / \mathrm{Ti}$ contacts are then evaporated at the edges of the Pt layer along the $y$ axis.

ISHE measurements have been performed in air at room temperature: spin-polarized electrons are excited by a focused beam (spot size $d \approx 10 \mu \mathrm{m}$ ) from a Ti:sapphire tunable laser, which provides photons in the 1.2-1.8 eV energy range. The circular polarization of the light is modulated by a photoelastic modulator (PEM) at $50 \mathrm{kHz}$ and the voltage difference $\Delta V$ between the two $\mathrm{Au} / \mathrm{Ti}$ electrodes, separated by about $3.5 \mathrm{~mm}$, is detected by a lock-in amplifier under open-circuit conditions. The wave-vector of the light inside the semiconductor, which sets the direction of the spin-polarization vector $\mathbf{P}$, can be continuously varied by changing the polar angle $\theta$ and the azimuthal angle $\varphi$, defined in Fig. 17.

Literature reports indicate that in $\mathrm{Si}$ the hole spin lifetime lies in the ps range 20, 21, 40, and is therefore longer than what reported for GaAs [41] and Ge [42]. Nevertheless, since the electron spin lifetime still exceeds by orders of magnitude this temporal range 24 29, the spin signal can be assumed to be mostly related to photogenerated electrons. This means that, while no charge current is injected or extracted into the semiconductor during our experiments, the two electron spin populations are unbalanced, thus yielding a pure spin current [35. Photogenerated electrons then diffuse towards the $\mathrm{Pt} / \mathrm{Si}$ interface: a Schottky barrier height of $E_{\mathrm{B}} \approx 0.85$ $\mathrm{eV}$ has been measured, in agreement with the results of Ref. 39 (see Fig. 1k) thus giving a a depletion region width $w_{\text {dep }}=1.1 \mu \mathrm{m}$. When the sample is illuminated under open-circuit conditions, the photon flux $\Phi$ lowers the Schottky barrier height (see Fig. 11p). Then, electrons are injected into the Pt layer via thermionic emission, carrying a spin current density $\mathbf{J}_{\mathrm{s}}$, which is converted into an effective electromotive field $E_{\text {ISHE }}$ yielding a voltage difference $\Delta V=E_{\mathrm{ISHE}} / l_{\mathrm{x}}$ between the two $\mathrm{Au} / \mathrm{Ti}$ electrodes [31, 37, where $\mathbf{E}_{\mathrm{ISHE}}=D_{\mathrm{ISHE}} \cdot\left(\mathbf{J}_{\mathrm{S}} \times \mathbf{P}\right)$, being $D_{\text {ISHE }}$ the efficiency of the ISHE process [30].

The spin-related nature of the detected signal has been verified by varying the projection of the spin polarization vector $\mathbf{P}$ along the $x$ axis. Fig. 22 shows the dependence of the voltage difference $\Delta V$ as a function of the angle $\theta$ for $\varphi=0^{\circ}$, an incident photon energy $h \nu=1.37 \mathrm{eV}$ and an incident power $W=1 \mathrm{~mW}$. The error bars take into account the $\Delta V$ variations between different datasets, originating from possible optical misalignements. A multilayer optical analysis on the $\mathrm{Pt} / \mathrm{Si}$ junction indicates that $\Delta V(\theta, \varphi) \propto t_{\mathrm{s}} t_{\mathrm{p}} \cos \delta \cos \varphi \tan \theta$ [43], where $t_{\mathrm{s}(\mathrm{p})}$ is the transmission coefficient of the $s(p)$-polarized light and $\delta$ is the angle between the direction of light propagation inside $\mathrm{Si}$ and the normal to the sample surface. Referring to Fig. 2a, we can set $\varphi=0^{\circ}$ : from the transmission coefficient values at $h \nu=1.37 \mathrm{eV}$ of Refs. 44 and 45 for $\mathrm{Pt}$ and $\mathrm{Si}$, respectively, it is possible to calculate the $\theta$-dependence of $\Delta V$ (red dashed line in Fig. $2 \mathrm{a}$ ). Such calculations are in good agreement with the experimental data thus confirming the spin-related origin of the measured signal. We have also investigated the behaviour of the ISHE signal as a function of the angle $\varphi$ for fixed $\theta=65^{\circ}$, as shown in Fig. 2b: the $\Delta V \propto \cos \varphi$ curve (red dashed line) fairly fits the experimental data, further confirming the spin-related nature of the detected signal.

The maximum absolute value of the ISHE signal, corresponding to $\Delta V=3.5 \pm 1.2 \mu \mathrm{V}$ is obtained for $\theta= \pm 65^{\circ}$ and it is comparable with the one detected in $\mathrm{Pt} / \mathrm{Ge}$ junctions under the same experimental conditions [37]. According to the theoretical calculations of Ref. 12, phonon-assisted optical transitions for $h \nu=$ $1.37 \mathrm{eV}$ should generate a net electron spin polarization $P \approx 0.7 \%$ (see Fig. 1p and below, Fig. 4p).

The $\Delta V$ dependence on the degree of circular polarization of the incident light $\left(P_{\text {circ }}\right)$ is reported in Fig. 3 a for $h \nu=1.37 \mathrm{eV}$ and $W=1 \mathrm{~mW}$ : in this case the measurements have been performed by varying the phase retardation $\Delta \xi$ of the PEM. Indeed, the temporal dependence of $P_{\text {circ }}$ can be expressed as $P_{\text {circ }}=-\sin [\Delta \xi \cos (\omega t)]$ : then the Fourier series expansion, truncated to the first harmonic, yields $P_{\text {circ }} \approx-2 J_{1}(\Delta \xi) \cos (\omega t)$, where $J_{1}(\Delta \xi)$ is the Bessel function of the first order. The experimental data of Fig. 33 reproduce the expected behaviour of $\Delta V$ as a function of $P_{\text {circ }}$.

The power dependence of the ISHE signal mirrors the behaviour of the photovoltage across a metal/semiconductor Schottky contact 37. It is monotonically increasing for incident powers below $\approx 1 \mathrm{~mW}$, 

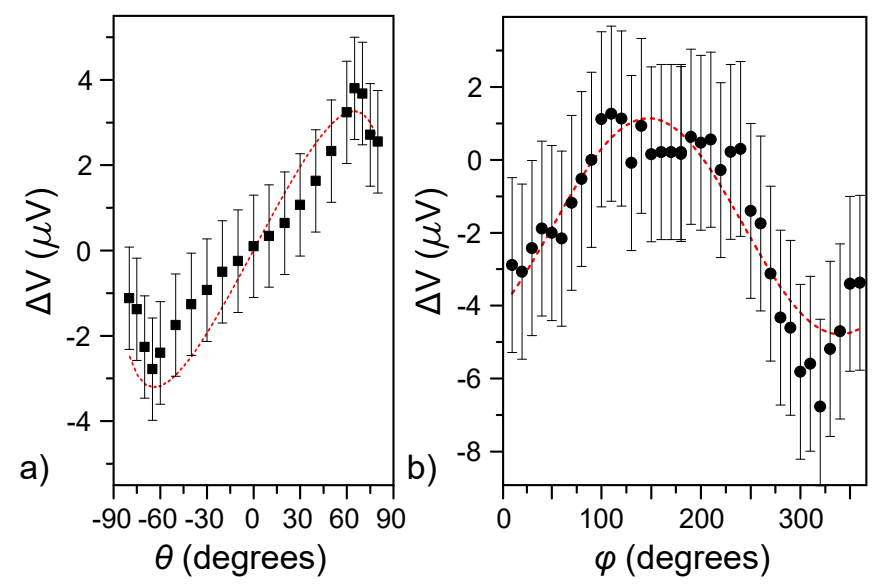

FIG. 2. (Color online) ISHE signal dependence as a function of (a) the angle $\theta$ for fixed $\varphi=0^{\circ}$ and (b) of the angle $\varphi$ for a fixed $\theta=65^{\circ}$. The measurements have been performed with a photon energy $h \nu=1.37 \mathrm{eV}$ and an incident power $W=$ $1 \mathrm{~mW}$. The red dashed line corresponds to the $\cos \alpha \tan \theta$ dependence (a) and $\cos \varphi$-dependence (b), obtained from a multilayer optical analysis of the $\mathrm{Pt} / \mathrm{Si}$ junction.

and saturates at higher illumination intensities.

In Fig. 4a, we report the photon energy dependence of the ISHE signal (red circles) in the 1.2-1.8 eV energy range, normalized to the incident photon flux $\Phi . \Delta V$ increases with $h \nu$ up to $h \nu \approx 1.4 \mathrm{eV}$, where we detect a broad maximum and then it decreases. The spectral evolution of the experimental data can be interpreted in the frame of a one-dimensional spin drift-diffusion model 2]. We define inside the semiconductor the spin density $s=n_{\uparrow}-n_{\downarrow}$ and the spin current density $J_{\mathrm{s}}=q\left(J_{\uparrow}-\right.$ $J_{\downarrow}$ ), where $J_{\uparrow(\downarrow)}$ are the spin-up(down) current densities related to the photo-generated spin-polarized electrons, respectively. Then the spatial distribution of $s$ and $J_{\mathrm{s}}$ can be described through the equations

$$
\begin{aligned}
\frac{1}{q} J_{\mathrm{s}}(x) & =-D_{\mathrm{n}} \frac{\partial s(x)}{\partial x}-\mu_{\mathrm{n}} s(x) E(x) \\
\frac{1}{q} \frac{\partial J_{\mathrm{s}}(x)}{\partial x} & =-\frac{s(x)}{\tau_{\mathrm{s}}}-w(x) s(x) p(x)+P \Phi \alpha e^{-\alpha x}
\end{aligned}
$$

The electric field $E(x)$, hole density $p(x)$ and the intrinsic generation-recombination rate $w(x)$ are obtained by numerically solving the coupled Poisson drift-diffusion equations [46] for electrons and holes photogenerated inside the semiconductor by the photon flux $\Phi$. For the electron mobility $\mu_{\mathrm{n}}=1380 \mathrm{~cm}^{2} \mathrm{Vs}^{-1}$, the electron diffusion coefficient $D_{\mathrm{n}}=\mu_{\mathrm{n}} k_{\mathrm{B}} T$ (being $k_{\mathrm{B}}$ and $T$ the Boltzmann constant and the temperature, respectively) and Shockley-Read-Hall recombination parameters, we have used the typical values of micro-eletronic device simulators [4]. Then, the photon energy dependent-absorption
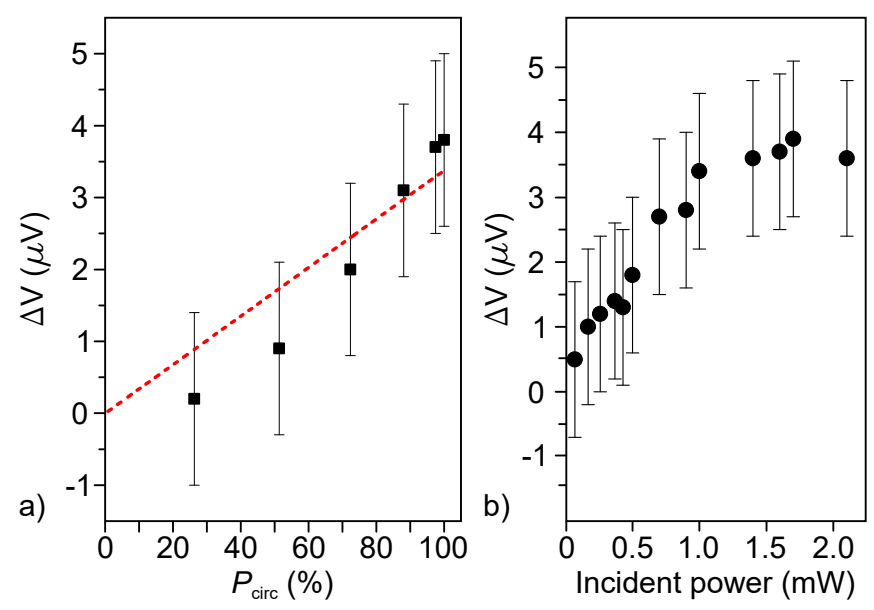

FIG. 3. (Color online) (a) ISHE signal dependence as a function of the degree of circular polarization of the light $\left(P_{\text {circ }}\right)$, for an incident photon energy of $h \nu=1.37 \mathrm{eV}$ and an incident power $W=1 \mathrm{~mW}$. The red dashed line highlights the linear behaviour of $\Delta V$. (b) $\Delta V$ dependence as a function of $W$ for an incident photon energy of $h \nu=1.37 \mathrm{eV}$.

coefficient $\alpha$ and initial degree of electron spin polarization $P$ are extracted from Refs. 45 and 12 , respectively, and reported in the inset of Fig. 4a.

At room temperature and relatively low doping densities, electron-phonon interactions set the momentum relaxation time $\tau_{\mathrm{p}}$ for $\Delta$ valley electrons [48. In this case, the electron spin lifetime $\tau_{\mathrm{s}}$ is dominated by the Yafet-Elliot mechanism and can therefore be expressed as $\tau_{\mathrm{s}}\left(E_{\mathrm{k}}\right)^{-1} \propto\left[\Delta_{\mathrm{so}} /\left(E_{\mathrm{g}}+\Delta_{\mathrm{so}}\right)\right]^{2}\left(E_{\mathrm{k}} / E_{\mathrm{g}}\right)^{2} \tau_{\mathrm{p}}\left(E_{\mathrm{k}}\right)^{-1}[2$, where $E_{\mathrm{k}}$ is the electron kinetic energy, $E_{\mathrm{g}}$ the energy gap and $\Delta_{\text {so }}$ the split-off energy. Therefore, in the case of $\mathrm{Si}, \tau_{\mathrm{s}}$ is expected to be approximately three orders of magnitude longer than $\tau_{\mathrm{p}}$. In $\operatorname{Si} \tau_{\mathrm{p}} \approx 30$ fs [12, while the energy relaxation time $\tau_{\text {en }}$, i.e. the average time required for hot electrons to thermalize at the bottom of the conduction band is $\tau_{\text {en }} \approx 260$ fs. Since $\tau_{\text {en }} \ll \tau_{\text {s }}$ most of the scattering events leading to spin relaxation will take place after electron thermalization and it is therefore a good approximation to consider $\tau_{\mathrm{s}}$ independent with respect to $E_{\mathrm{k}}$, i.e. on the photon energy $h \nu$. Then, by solving Eqs. 1 we can calculate the spin current density $J_{\mathrm{s} 0}$, proportional to the ISHE signal $\Delta V$, injected from the semiconductor into the $\mathrm{Pt}$ layer as a function of the incident photon energy with $\tau_{\mathrm{s}}$ as free fitting parameter for the experimental data of Fig. 4a.

Fig. $4 \mathrm{~b}$ shows the $h \nu$-dependence of the normalized $J_{\mathrm{s} 0}$ for $\tau_{\mathrm{s}}=5 \mathrm{~ns}, 25$ and $100 \mathrm{~ns}$ (black, red and blue continuous lines respectively in Fig. 4 4 b). The spectral evolution of $J_{\mathrm{s} 0}$ can be interpreted in terms of the interplay between the photon energy dependence of $\alpha$ and $P$, reported in the inset of Fig. 4 $4 \mathrm{~b}$. For $h \nu=1.18 \mathrm{eV}$, electrons are generated with a spin polarization $P=1.4 \%$ in a region relatively far from the $\mathrm{Pt} / \mathrm{Si}$ interface due to the low photon absorption rate. As a consequence, the 

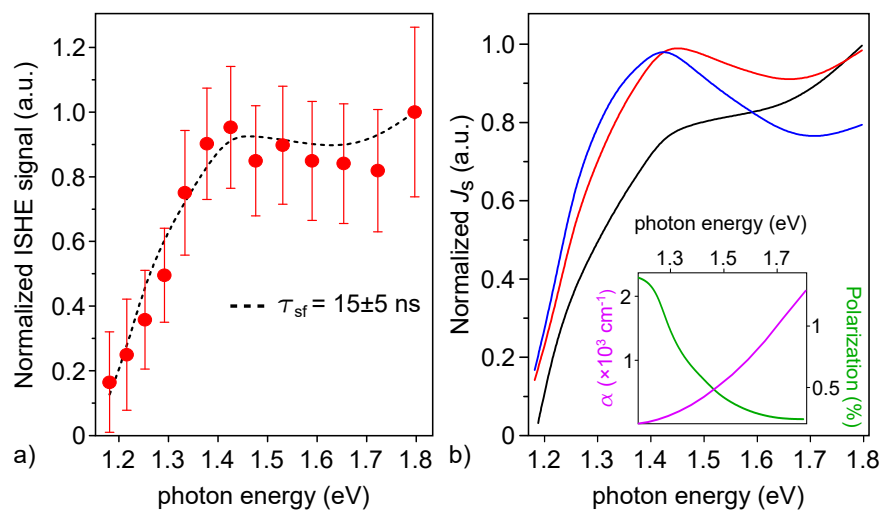

FIG. 4. (Color online) (a) ISHE signal, as a function of the incident photon energy, normalized with respect to the incident photon flux $\Phi$ (red dots). The black dashed curve identifies the fitting function obtained by solving Eqs. 1 for the best fit parameter $\tau_{\mathrm{s}}=15 \pm 5 \mathrm{~ns}$, which corresponds to the spin lifetime of electrons thermalized at the bottom of the Si conduction band. Inset: photon energy dependent-absorption coefficient $\alpha$ and initial degree of electron spin polarization $P$ from Refs. 45 and 12 respectively, as a function of the incident photon energy. (b) Normalized $h \nu$-dependence of $J_{\mathrm{s} 0}$ from Eqs. 1 for electron spin lifetimes $\tau_{\mathrm{s}}=5$ (black curve), 25 (red curve) and 100 ns (blue curve).

spin current at the $\mathrm{Pt} /$ semiconductor interface is relatively small. For $h \nu \rightarrow 1.8 \mathrm{eV}$, an increasing spin density is generated close to the $\mathrm{Pt}$ layer, and, despite the lower initial polarization, $J_{\mathrm{s} 0}$ increases. It is interesting to note that overall photon-energy dependence of $J_{\mathrm{s} 0}$ is relatively sensitive to the value of $\tau_{\mathrm{s}}$, qualitatively reproducing the experimental data of Fig. 4 a.

The best fit of the experimental photon energy dependence of Fig. 4a is thus obtained for $\tau_{\mathrm{s}}=15 \pm 5 \mathrm{~ns}$ (black dashed line), in good agreement with theoretical calculations reported in the literature [24, 48, 49]. The simple drift-diffusion model of Eqs. 1 nicely reproduce the experimental data unraveling the fundamental issues of spin diffusion at the $\Delta$ minima of Si: the spin current density $J_{\mathrm{s}}$ at the $\mathrm{Pt} / \mathrm{Si}$ interface is dictated by the trade-off between the absorption coefficient $\alpha$, which is very small in bulk $\mathrm{Si}$ especially close to the indirect bandgap, and the spin lifetime $\tau_{\mathrm{s}}$. Moreover, our experimental estimation of $\tau_{\mathrm{s}}$ corresponds to a spin diffusion length $L_{\mathrm{s}}=\sqrt{D_{\mathrm{n}} \tau_{\mathrm{s}}}=7 \pm 2 \mu \mathrm{m}$. Such a value exceeds the standard dimensions of common electronic building blocks and suggests that bulk Si can play a central role in the design of novel spintronic devices.

It is interesting to remark that the ISHE signal in $\mathrm{Pt} / \mathrm{Ge}$ and $\mathrm{Pt} / \mathrm{Si}$ junctions is of the same order of magnitude for similar Pt thicknesses. In bulk Ge, the optical orientation process can generate an initial spin polarization up to $P \approx 50 \%$ at the direct Ge bandgap [10, 50]. This value is then partially lowered by the ultrafast $\Gamma-L$ scattering which electrons undergo in the Ge conduction band [51]. In Si the spin current generation and diffusion both take place at the $\Delta$-valleys: although the initial spin polarization is much smaller, the larger spin lifetime $\left(\tau_{\mathrm{s}} \approx 1 \mathrm{~ns}\right.$ and $\approx 15 \mathrm{~ns}$ in bulk Ge and $\mathrm{Si}$, respectively, at room temperature and for lightly-doped samples [35, 52 54]) allows for an ISHE signal which is comparable to the one detected in bulk Ge.

It is also worth noticing that spin-polarized photoemission experiments at $\mathrm{T}=120 \mathrm{~K}$ in the same photon energy range, performed by the authors [55] on p-doped bulk $\mathrm{Si}(001)$ samples, did not reveal any spin-related signal within the experimental error. However, at variance from Mott polarimetry exploited in Ref. 55, where the efficiency of the spin-detector is around $10^{-4}$, photoinduced ISHE measurement can be performed by means lock-in based detection schemes, which greatly enhance its sensitivity, thus enabling the detection of very low spin polarization signals.

In conclusion, we have experimentally demonstrated optical spin orientation at the direct gap of $\mathrm{Si}$ at room temperature. The inverse spin-Hall effect in a Pt/Si junction have been used to detect spin current density generated for photon energies comprised between 1.2 and $1.8 \mathrm{eV}$. The photon energy dependence of the ISHE signal can be interpreted by means of spin drift-diffusion model which also provides an estimation of the electron spin lifetime $\tau_{\mathrm{s}}=15 \pm 5 \mathrm{~ns}$, in good agreement with theoretical calculations of Refs. 24, 48, and 49. These results lay the groundwork for spintronic devices based on bulk Si.

The authors would like to thank M. Jamet for a critical reading of the manuscript and fruitful discussions. Partial funding is acknowledged to the CARIPLO project SEARCH-IV (grant 2013-0623).

* federico.bottegoni@polimi.it

[1] F. Sze and B. P. Zakharchenya, eds., Optical orientation (Modern Problems in Condensed Matter Sciences, Vol. 8) (Elsevier, Amsterdam, 1984) p. 523.

[2] I. Zutić, J. Fabian, and S. Das Sarma, Rev. Mod. Phys. 76, 323 (2004), arXiv:0405528 [cond-mat].

[3] G. Lampel, Phys. Rev. Lett. 20, 491 (1968)

[4] M. S. Tyagi and R. Van Overstraeten, Solid State Electron. 26, 577 (1983).

[5] J. A. del Alamo and R. M. Swanson, Solid State Electron. 30, 1127 (1987).

[6] R. Parsons, Phys. Rev. Lett. 23, 1152 (1969).

[7] D. Pierce and F. Meier, Phys. Rev. B 13, 5484 (1976)

[8] F. Ciccacci, J. Appl. Phys. 53, 4395 (1982)

[9] R. Allenspach, F. Meier, and D. Pescia, Phys. Rev. Lett. $\mathbf{5 1 , 2 1 4 8 ( 1 9 8 3 )}$

[10] F. Bottegoni, G. Isella, S. Cecchi, and F. Ciccacci, Appl. Phys. Lett. 98, 242107 (2011)

[11] P. Li and H. Dery, Phys. Rev. Lett. 105, 3 (2010).

[12] J. L. Cheng, J. Rioux, J. Fabian, and J. E. Sipe, Phys. Rev. B 83, 165211 (2011). 
[13] N. Sircar and D. Bougeard, Phys. Rev. B 89, 041301 (2014)

[14] B. T. Jonker, G. Kioseoglou, A. T. Hanbicki, C. H. Li, and P. E. Thompson, Nat. Phys. 3, 542 (2007).

[15] I. Appelbaum, B. Huang, and D. J. Monsma, Nature 447, 295 (2007)

[16] B. Huang, D. J. Monsma, and I. Appelbaum, Phys. Rev. Lett. 99, 177209 (2007), arXiv:0706.0866

[17] O. M. J. van 't Erve, A. T. Hanbicki, M. Holub, C. H. Li, C. Awo-Affouda, P. E. Thompson, and B. T. Jonker, Appl. Phys. Lett. 91, 212109 (2007)

[18] C. H. Li, G. Kioseoglou, O. M. J. van t Erve, P. E. Thompson, and B. T. Jonker, Appl. Phys. Lett. 95, 172102 (2009)

[19] L. Grenet, M. Jamet, P. Noe, V. Calvo, J.-M. Hartmann, L. E. Nistor, B. Rodmacq, S. Auffret, P. Warin, and Y. Samson, Appl. Phys. Lett. 94, 032502 (2009)

[20] S. P. Dash, S. Sharma, R. S. Patel, M. P. de Jong, and R. Jansen, Nature 462, 491 (2009).

[21] E. Shikoh, K. Ando, K. Kubo, E. Saitoh, T. Shinjo, and M. Shiraishi, Phys. Rev. Lett. 110, 127201 (2013)

[22] A. Dankert, R. S. Dulal, and S. P. Dash, Sci. Rep. 3, 1 (2013).

[23] T. Sasaki, Y. Ando, M. Kameno, T. Tahara, H. Koike, T. Oikawa, T. Suzuki, and M. Shiraishi, Phys. Rev. Appl. 2, 034005 (2014)

[24] P. Li and H. Dery, Phys. Rev. Lett. 107, 1 (2011).

[25] H. Dery, Y. Song, P. Li, and I. Zutic, Appl. Phys. Lett. 99, $082502(2011)$.

[26] R. Jansen, Nat. Mater. 11, 400 (2012).

[27] J. Li, L. Qing, H. Dery, and I. Appelbaum, Phys. Rev. Lett. 108, 157201 (2012).

[28] O. Restrepo and W. Windl, Phys. Rev. Lett. 109, 1 (2012)

[29] Y. Song, O. Chalaev, and H. Dery, Phys. Rev. Lett. 113, 1 (2014)

[30] E. Saitoh, M. Ueda, H. Miyajima, and G. Tatara, Appl. Phys. Lett. 88, 182509 (2006)

[31] K. Ando, M. Morikawa, T. Trypiniotis, Y. Fujikawa, C. H. W. Barnes, and E. Saitoh, Appl. Phys. Lett. 96, $082502(2010)$

[32] F. Bottegoni, A. Ferrari, G. Isella, M. Finazzi, and F. Ciccacci, Phys. Rev. B 88, 121201 (2013)

[33] G. Isella, F. Bottegoni, A. Ferrari, M. Finazzi, F. Ciccacci, G. Isella, F. Bottegoni, A. Ferrari, M. Finazzi, and F. Ciccacci, Appl. Phys. Lett. 106, 232402 (2015).

[34] S. K. Khamari, S. Porwal, V. K. Dixit, and T. K. Sharma, Appl. Phys. Lett. 104, 042102 (2014).

[35] F. Bottegoni, A. Ferrari, S. Cecchi, M. Finazzi, F. Ciccacci, and G. Isella, Appl. Phys. Lett. 102, 152411 (2013)
[36] F. Bottegoni, M. Celebrano, M. Bollani, P. Biagioni, G. Isella, F. Ciccacci, and M. Finazzi, Nat. Mater. 13, 790 (2014)

[37] F. Bottegoni, A. Ferrari, F. Rortais, C. Vergnaud, A. Marty, G. Isella, M. Finazzi, M. Jamet, and F. Ciccacci, Phys. Rev. B 92, 214403 (2015).

[38] G. F. Koster, J. O. Dimmock, W. R. G., and H. Statz, eds., The Properties of the Thirty-Two Point Groups (MIT Press, Cambridge, MA, 1963).

[39] H. K. Liou, X. Wu, U. Gennser, V. P. Kesan, S. S. Iyer, K. N. Tu, and E. S. Yang, Appl. Phys. Lett. 60, 577 (1992)

[40] K. Ando and E. Saitoh, Nat. Commun. 3, 629 (2012).

[41] J. Kikkawa and D. Awschalom, Phys. Rev. Lett. 80, 4313 (1998).

[42] E. J. Loren, J. Rioux, C. Lange, J. E. Sipe, H. M. van Driel, and A. L. Smirl, Phys. Rev. B 84, 214307 (2011).

[43] K. Ando, M. Morikawa, T. Trypiniotis, Y. Fujikawa, C. H. W. Barnes, and E. Saitoh, J. Appl. Phys. 107, $113902(2010)$

[44] A. D. Rakić, A. B. Djurišić, J. M. Elazar, and M. L. Majewski, Appl. Opt. 37, 5271 (1998).

[45] M. A. Green and M. J. Keevers, Prog. Photovoltaics Res. Appl. 3, 189 (1995)

[46] S. Birner, T. Zibold, T. Andlauer, T. Kubis, M. Sabathil, A. Trellakis, and P. Vogl, IEEE Trans. Electron Devices 54, 2137 (2007).

[47] R. Klix, W Dittmann and R. Stenzel, Lecture Notes in Computer Science (Springer-Verlag, 1994) pp. 99-104.

[48] Y. Song and H. Dery, Phys. Rev. B 86, 1 (2012).

[49] J. L. Cheng, M. W. Wu, and J. Fabian, Phys. Rev. Lett. 104, 1 (2010)

[50] J. Rioux and J. E. Sipe, Phys. Rev. B 81, 155215 (2010).

[51] F. Pezzoli, F. Bottegoni, D. Trivedi, F. Ciccacci, A. Giorgioni, P. Li, S. Cecchi, E. Grilli, Y. Song, M. Guzzi, H. Dery, and G. Isella, Phys. Rev. Lett. 108, 156603 (2012).

[52] C. Guite and V. Venkataraman, Appl. Phys. Lett. 101, 252404 (2012)

[53] P. Li, Y. Song, and H. Dery, Phys. Rev. B 86, 085202 (2012)

[54] F. Rortais, S. Oyarzún, F. Bottegoni, J.-C. RojasSánchez, P. Laczkowski, A. Ferrari, C. Vergnaud, C. Ducruet, C. Beigné, N. Reyren, A. Marty, J.-P. Attané, L. Vila, S. Gambarelli, J. Widiez, F. Ciccacci, H. Jaffrès, J.-M. George, and M. Jamet, J. Phys. Condens. Matter 28, 165801 (2016)

[55] a. Ferrari, F. Bottegoni, G. Isella, S. Cecchi, and F. Ciccacci, Phys. Rev. B 88, 115209 (2013). 